\title{
DETERMINAÇÃO DO TEOR DE CÁLCIO EM COMPRIMIDO À BASE DE LACTATO DE CÁLCIO UTILIZADO NO TRATAMENTO DA OSTEOPOROSE
}

Sheila P. M. C. de Souza, Francisca E. de Morais, Elisama V. dos Santos, Márcia L. da Silva, Carlos A. Martinez-Huitle e Nedja S. Fernandes*

Instituto de Química, Universidade Federal do Rio Grande do Norte, Av. Senador Salgado Filho, 3000, Campus Universitário, 59078-970 Natal - RN, Brasil

Recebido em 20/9/11; aceito em 9/3/12; publicado na web em 2/7/12

\begin{abstract}
DETERMINATION OF CALCIUM IN CALCIUM LACTATE TABLETS USED IN THE TREATMENT OF OSTEOPOROSIS. The present study utilized thermogravimetry (TG) and optical emission spectrometry with inductively coupled plasma (ICP/OES) to determine the amount of calcium in calcium lactate tablets used in the treatment of osteoporosis. Thermogravimetry results indicated that the decomposition temperature of $\mathrm{CaCO}_{3}$ occurred at 603.9 and $609.4{ }^{\circ} \mathrm{C}$ in the samples of calcium lactate and tablets, respectively. The calcium content obtained by TG for the tablets sample showed a similar result to that disclosed by ICP-OES, indicating a value of $8.93 \%$ for both techniques.
\end{abstract}

Keywords: termogravimetry; calcium; osteoporosis.

\section{INTRODUÇÃO}

A osteoporose é definida como uma redução da massa óssea acompanhada pela degeneração da sua qualidade, resultando numa redução da resistência mecânica do osso e, portanto, um risco maior de fraturas. ${ }^{1}$ É uma doença que se manifesta na fase tardia da vida, podendo ter origem durante o crescimento do esqueleto. É um problema de saúde verificado em todas as partes do mundo. A partir dos 50 anos, $30 \%$ das mulheres e $13 \%$ dos homens poderão sofrer algum tipo de fratura, estimando-se que a incidência de fraturas irá quadruplicar nos próximos 50 anos, em decorrência do aumento da expectativa de vida. ${ }^{2}$

A Sociedade Brasileira de Endocrinologia e Metabologia declara a não existência de dados exatos sobre a incidência da osteoporose no Brasil, mas considera que aproximadamente 10 milhões de pessoas sofrem com a doença, a grande maioria com idade acima de 60 anos. ${ }^{3}$

Dois nutrientes, cálcio e vitamina $\mathrm{D}$, são especialmente necessários para se ter ossos fortes. O cálcio é essencial para a manutenção óssea, enquanto o papel da vitamina D é ajudar na absorção do cálcio e na manutenção da densidade óssea.

No início, o tratamento da osteoporose deve ser realizado através da suplementação de cálcio e vitamina $\mathrm{D}$, utilizando-se diferentes sais de cálcio. O mais indicado é o carbonato de cálcio, pois apresenta uma maior quantidade de cálcio elementar, da ordem de $40 \%$. O citrato de cálcio apresenta $21 \%$ de cálcio elementar e o lactato de cálcio $13 \%$. Aconselha-se a reposição de $500 \mathrm{mg}$ a $1 \mathrm{~g}$ por dia, devendo ser ingerido às refeições, para facilitar a absorção. ${ }^{2}$

O lactato de cálcio é um composto à base de cálcio, produzido quando o ácido lático reage com carbonato de cálcio. A reação forma cristais brancos que estão presentes no leite e em outros produtos lácteos. Apresenta níveis mais elevados de solubilidade no corpo, tornando mais fácil a absorção do cálcio no intestino. ${ }^{4}$

O lactato de cálcio, Figura $1 \mathrm{~S}$ do material suplementar, é um suplemento alimentar empregado no tratamento e prevenção da deficiência de cálcio e pode ser utilizado em indivíduos que não recebem os níveis de cálcio suficientes através da ingestão de alimentos. Também pode ser útil no tratamento de problemas médicos que resultam de não se ter quantidade suficiente de cálcio, incluindo

*e-mail:nedja@ufrnet.br ossos enfraquecidos (conhecido como raquitismo ou osteomalacia), osteoporose (perda de massa óssea) e tetania latente (um distúrbio muscular). É geralmente encontrado como suplemento de cálcio e, mais frequentemente, prescrito para mulheres grávidas.

Os medicamentos à base de lactato de cálcio são os que fornecem menor teor de cálcio elementar por comprimido, por isso recomenda-se fazer a ingestão de pelo menos um comprimido de 4 a 6 vezes ao dia, para que se atenda às necessidades diárias de reposição. Além disso, por ser um sal derivado do ácido lático esse tipo de medicamento é mais recomendado para mulheres grávidas e crianças até os 10 anos.

Os medicamentos vêm sendo cada vez mais objetos de preocupação e de inúmeras pesquisas realizadas mundialmente. Estas dizem respeito à prática médica, reações adversas, aumento e disseminação de resistência bacteriana a antibióticos, padrão e influência da propaganda na prescrição de medicamentos. ${ }^{5}$

A determinação dos princípios ativos, através dos procedimentos convencionais, em alguns casos, torna-se difícil, havendo a necessidade de isolar o componente ativo. Dessa forma, é necessário utilizar novas técnicas que forneçam informações mais rápidas e eficientes, possibilitando uma nova rotina no procedimento analítico dos fármacos. As técnicas termoanalíticas (TG, DSC e DTA) vêm se caracterizando como ferramentas importantes e bastantes eficazes, pois são capazes de produzir resultados rápidos e reprodutíveis. ${ }^{6}$

Dessa forma, o presente trabalho se propôs a realizar um estudo termoanalítico nos medicamentos utilizados para combater a osteoporose a partir do uso da termogravimetria (TG), análise térmica diferencial (DTA) e calorimetria exploratória diferencial (DSC) e comparar os resultados obtidos em relação ao teor de cálcio, empregando termogravimetria e espectrometria de emissão óptica com plasma indutivamente acoplado (ICP-OES).

$\mathrm{Na}$ literatura encontra-se relato sobre o estudo do comportamento térmico do lactato de cálcio penta-hidratado. A curva TG desse sal apresenta uma etapa de desidratação e posterior formação de um composto intermediário que se decompõe para formar o carbonato de cálcio. Na sequência, ocorre a decomposição térmica do carbonato de cálcio, com formação de $\mathrm{CaO}$. Nesse trabalho também foram quantificados o teor de água, com um percentual de $28,5 \%$, e o teor de $18,5 \%$ de $\mathrm{CaO}^{7}$ 
O comportamento da desidratação e da hidratação do lactato de cálcio penta-hidratado e anidro foram investigados por meio da TG, DSC, difração de raios x (DRX) e microscopia eletrônica de varredura (MEV). ${ }^{8}$ Os resultados mostraram que a desidratação do lactato de cálcio penta-hidratado resulta em uma completa transformação para uma amostra não cristalina, com mudanças significativas no tamanho da partícula e no estado cristalino. Por outro lado, $75 \%$ da amostra anidra ao ser hidratada apresentou cristalinidade.

$\mathrm{O}$ efeito da pulverização e secagem de medicamentos à base de lactato de cálcio penta-hidratado foi estudado utilizando as técnicas de TG/DTA, DSC e DRX. ${ }^{9}$ Os resultados obtidos indicaram que à medida que ocorria a desidratação, eram observadas modificações nas propriedades farmacêuticas do comprimido, tais como, dureza, espessura e diâmetro. Estes resultados sugerem que as mudanças nessas propriedades são fortemente dependentes da cinética de desidratação; por outro lado, a desidratação leva a uma maior agregação entre as partículas do lactato de cálcio.

O uso da termogravimetria na determinação do teor de cálcio em cascas de ovos de galinha e codorna foi investigado. ${ }^{10}$ Os resíduos obtidos a partir da termogravimetria foram caracterizados por espectroscopia de absorção na região do infravermelho, comprovando-se através dos espectros que eram constituídos basicamente por óxido de cálcio. Para comparação dos resultados foram realizadas análises por fotometria de chama e titulação complexométrica, mostrando resultados satisfatórios e comprovando a eficácia da termogravimetria na determinação do teor de cálcio.

\section{PARTE EXPERIMENTAL}

O lactato de cálcio e a amostra de comprimido foram adquiridos no comércio local. Em seguida, foram pesados, pulverizados em gral de ágata, acondicionados em frascos de vidro âmbar e armazenados em dessecador para realização das análises. A Tabela 1 apresenta as informações sobre a composição da amostra utilizada.

Para a análise de cálcio por ICP-OES pesaram-se $500 \mathrm{mg}$ de cada amostra em uma cápsula de porcelana e calcinou-se até $600{ }^{\circ} \mathrm{C}$ por $4 \mathrm{~h}$. Em seguida, adicionou-se $\mathrm{HNO}_{3}$ concentrado até a total dissolução das amostras e completou-se o volume de $100 \mathrm{~mL}$ com água deionizada, sendo esse procedimento realizado em triplicata.

\section{Espectroscopia de absorção na região do infravermelho (IV)}

Os espectros de absorção na região do infravermelho foram obtidos em um espectrofotômetro infravermelho Thermo Nicolet, modelo Nexus 470 FTIR, utilizando-se pastilhas de $\mathrm{KBr}$, sendo as análises realizadas na região de $4000 \mathrm{a} 400 \mathrm{~cm}^{-1}$.

\section{Termogravimetria/termogravimetria derivada (TG/DTG)}

As análises termogravimétricas foram obtidas em um sistema Shimadzu, modelo TGA-50 e DTG-60, que opera da temperatura ambiente até $1000^{\circ} \mathrm{C}$, nas seguintes condições: faixa de temperatura de 25 a $900{ }^{\circ} \mathrm{C}$, razão de aquecimento de $10^{\circ} \mathrm{C} \mathrm{min}^{-1}$, atmosfera de ar

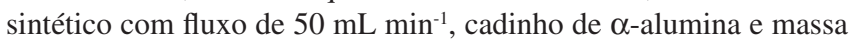
de aproximadamente $7 \mathrm{mg}$.

\section{Análise térmica diferencial (DTA)}

A análise térmica diferencial foi realizada em um sistema acoplado (TG/DTA) da Shimadzu, modelo DTG-60, capaz de operar da temperatura ambiente até $1500^{\circ} \mathrm{C}$, nas mesmas condições descritas acima.

\section{Calorimetria exploratória diferencial (DSC)}

As curvas DSC foram obtidas em um sistema Shimadzu, modelo DSC $50 \mathrm{H}$, capaz de operar da temperatura ambiente até $725^{\circ} \mathrm{C}$, controlado por um microprocessador e nas seguintes condições: faixa de temperatura de 25 a $500{ }^{\circ} \mathrm{C}$, razão de aquecimento de $10^{\circ} \mathrm{C}$ $\mathrm{min}^{-1}$, atmosfera de nitrogênio com fluxo de $50 \mathrm{~mL} \mathrm{~min}^{-1}$, cadinho de alumínio e massa de aproximadamente $2 \mathrm{mg}$ de amostra. A célula calorimétrica foi calibrada utilizando-se o metal índio de pureza $99,99 \%$ com $\mathrm{TF}=156,4^{\circ} \mathrm{C}$ e $\mathrm{DH}_{\text {fus. }}=6,6 \mathrm{cal} \mathrm{g}^{-1}$.

\section{Difração de raios X (DRX)}

A avaliação da cristalinidade do princípio ativo e da forma farmacêutica, além dos compostos intermediários obtidos a partir da decomposição térmica a $550{ }^{\circ} \mathrm{C}$ e dos resíduos obtidos a $900{ }^{\circ} \mathrm{C}$ pela termogravimetria, foi realizada em um difratômetro de raios $\mathrm{X}$, modelo XDR-6000, da Shimadzu, utilizando uma fonte de radiação de $\mathrm{CuK} \alpha$ e velocidade de varredura de $2 \theta \mathrm{min}^{-1}$ pelo método de pó.

\section{Espectrometria de emissão óptica com plasma indutivamente} acoplado (ICP-OES)

As análises de cálcio foram realizadas no Núcleo de Processamento Primário e Reuso de Água produzida e Resíduo, NUPPRAR, da UFRN utilizando um espectrômetro de emissão óptica com plasma indutivamente acoplado, modelo ICAP 6300 da Shimadzu, utilizando o método USEPA 6010C (United States Environmental Protection Agency).

\section{Microscopia eletrônica de varredura (MEV)}

As micrografias das amostras foram obtidas em um microscópio eletrônico de varredura, modelo Philips XL-30. Para a obtenção das imagens as condições instrumentais foram: $20 \mathrm{~V}$, diâmetro do feixe 4,0 (aumento de acordo com a necessidade de cada amostra), o detector utilizado foi retroespalhado.

\section{RESULTADOS E DISCUSSÃO}

Os espectros de absorção na região do infravermelho estão apresentados na Figura 2S, material suplementar. $\mathrm{Na}$ amostra de lactato de cálcio verifica-se em $3235 \mathrm{~cm}^{-1}$ uma banda larga referente ao estiramento simétrico da ligação O-H existente na estrutura química do fármaco, como também devida à presença de moléculas de água. A banda na região de $2352 \mathrm{~cm}^{-1}$ pode ser atribuída ao estiramento simétrico da ligação $\mathrm{C}-\mathrm{H}$ e as bandas de absorção relacionadas aos estiramentos assimétrico e simétrico da ligação $\mathrm{C}=\mathrm{O}$ aparecem, respectivamente, em 1592 e $1412 \mathrm{~cm}^{-1}$.

Tabela 1. Informações sobre a amostra do comprimido incluindo quantidade do princípio ativo, excipientes e médias das massas das amostras

\begin{tabular}{cccc}
\hline Amostra & Princípio ativo & Excipientes & $\begin{array}{c}\text { Média das massas do compri- } \\
\text { mido (mg)/desvio padrão }\end{array}$ \\
\hline Comprimido & $\begin{array}{c}\text { Lactato de cálcio } \\
(45,83 \text { mg de cálcio } \\
\text { elementar })\end{array}$ & $\begin{array}{c}\text { Amido de milho, talco farma- } \\
\text { cêutico, estearato de magnésio, } \\
\text { celulose microcristalina e lauril } \\
\text { sulfato de sódio }\end{array}$ & $348,7 \pm 6,88$ \\
\hline
\end{tabular}


$\mathrm{Na}$ amostra do comprimido observa-se na região de $3406 \mathrm{~cm}^{-1}$ uma banda larga referente ao estiramento simétrico da ligação $\mathrm{O}-\mathrm{H}$ existente na estrutura química do princípio ativo e a presença de moléculas de água, conforme mostrado na curva termogravimétrica. A banda que pode ser atribuída ao estiramento simétrico da ligação C-H encontra-se deslocada em relação ao espectro do princípio ativo para o valor de $2931 \mathrm{~cm}^{-1}$. Observa-se um deslocamento também nos valores das bandas de absorção relacionadas aos estiramentos assimétrico e simétrico da ligação $\mathrm{C}=\mathrm{O}$ que aparecem, respectivamente, em 1630 e 1393 cm$^{-1}$; esses deslocamentos podem ser atribuídos à presença dos diferentes excipientes que fazem parte da formulação da amostra de comprimido.

A Figura 1 mostra as curvas TG/DTG do lactato de cálcio (princípio ativo) e da amostra do comprimido. Na curva TG/DTG do lactato de cálcio, observam-se cinco etapas de perdas de massa, sendo a primeira entre 47,4 e $134,5^{\circ} \mathrm{C}\left(\mathrm{DTG}_{\mathrm{PICO}}=99,5^{\circ} \mathrm{C}\right)$ atribuída à desidratação com liberação de 5 mols de água, correspondendo a uma perda de massa de $25,3 \%$, com formação posterior do lactato de cálcio anidro. Esses resultados estão em acordo com os obtidos por Wesolowski e Konarski, ${ }^{7}$ ao investigarem a decomposição térmica de alguns sais de cálcio utilizados em medicina. Uma segunda etapa de decomposição ocorre no intervalo entre 266,1 a $335,4{ }^{\circ} \mathrm{C}\left(\mathrm{DTG}_{\mathrm{PICO}}=\right.$ $302,8^{\circ} \mathrm{C}$ ) com um percentual de $9,9 \%$ de perda de massa decorrente da decomposição do lactato de cálcio; a terceira e quarta etapas de decomposição ocorrem de forma consecutiva, sendo a terceira no intervalo entre 386,8 a $415,6^{\circ} \mathrm{C}\left(\mathrm{DTG}_{\mathrm{PICO}}=407,5^{\circ} \mathrm{C}\right)$ e a quarta na faixa de 415,6 a $435,7{ }^{\circ} \mathrm{C}\left(\mathrm{DTG}_{\mathrm{PICO}}=428,6^{\circ} \mathrm{C}\right)$ com a formação de $\mathrm{CaCO}_{3}$. A quinta etapa de decomposição ocorre entre 603,9 a $725,2^{\circ} \mathrm{C}$ $\left(\mathrm{DTG}_{\mathrm{PICO}}=705,2^{\circ} \mathrm{C}\right) \mathrm{com}$ um percentual de $14,4 \%$ de perda de massa, correspondente à decomposição do carbonato de cálcio e formação do óxido de cálcio. A partir da curva TG os cálculos indicaram um percentual de pureza de $94,9 \%$ do lactato de cálcio e de 13,0\% de cálcio elementar para essa amostra.

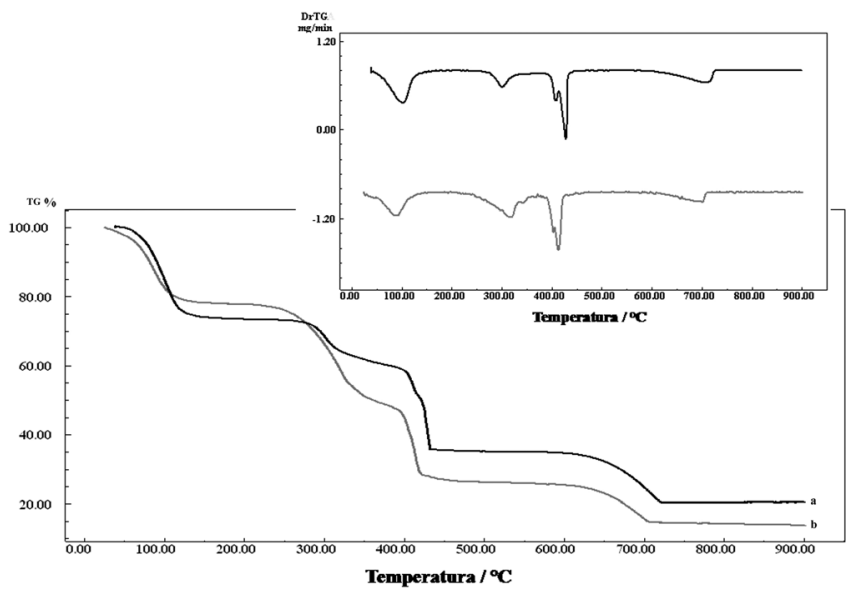

Figura 1. Curvas TG/DTG de (a) lactato de cálcio e (b) amostra do comprimido

Na curva TG/DTG da amostra do comprimido, observam-se cinco etapas de perda de massa, sendo a primeira no intervalo de 29,3 a $133,9^{\circ} \mathrm{C}\left(\mathrm{DTG}_{\mathrm{PICO}}=98,0^{\circ} \mathrm{C}\right)$ que pode ser atribuída à desidratação da amostra, correspondendo a uma perda de massa de 20,6\%. A segunda e terceira etapas ocorrem consecutivamente, sendo que a segunda é visualizada no intervalo entre 240,0 a $375,5^{\circ} \mathrm{C}\left(\mathrm{DTG}_{\mathrm{PICO}}\right.$ $=317,7^{\circ} \mathrm{C}$ ) $\operatorname{com} 28,9 \%$ de perda de massa e pode estar relacionada à formação de compostos intermediários da decomposição térmica do lactato de cálcio e dos excipientes amido de milho, estearato de magnésio, celulose microcristalina e lauril sulfato de sódio. A celulose microcristalina sofre decomposição entre 285,0 e $350,0{ }^{\circ} \mathrm{C}$ com perda de massa de $76,0 \% .{ }^{11}$ A terceira e quarta etapas ocorrem consecutivamente no intervalo entre 375,5 a $428,5{ }^{\circ} \mathrm{C}\left(\mathrm{DTG}_{\mathrm{PICO}}=\right.$ 409,9 e $419,8^{\circ} \mathrm{C}$ ) com perda de massa total de $20,1 \%$ e formação de $\mathrm{CaCO}_{3}$. A última etapa de perda de massa, que ocorre entre 609,4 a $721,7^{\circ} \mathrm{C}\left(\mathrm{DTG}_{\mathrm{PICO}}=714,4^{\circ} \mathrm{C}\right)$, com um percentual de $9,5 \%$ é atribuída à decomposição do $\mathrm{CaCO}_{3}$. A partir dessa informação observa-se a formação de $17,0 \%$ de resíduo final constituído por $\mathrm{CaO}$ e $\mathrm{MgO}$, este proveniente da decomposição do estearato de magnésio. Estes óxidos não se decompõem até $900{ }^{\circ} \mathrm{C}$, assim como o talco que é bastante estável termicamente.

A Figura 2 apresenta as curvas DTA do lactato de cálcio e da amostra de comprimido. Na curva DTA do lactato de cálcio, podem-se observar dois picos endotérmicos, o primeiro entre 59,7 a $118,3{ }^{\circ} \mathrm{C}$ e o segundo no intervalo de 118,3 a $161,3{ }^{\circ} \mathrm{C}$, indicativos do processo de desidratação. Verifica-se ainda um pico exotérmico entre 390,9 e $453,4{ }^{\circ} \mathrm{C}$, que pode estar relacionado à decomposição do produto intermediário do lactato de cálcio anidro em $\mathrm{CaCO}_{3}$, e uma endoterma entre 647,4 e $723,2^{\circ} \mathrm{C}$, atribuída à decomposição térmica do $\mathrm{CaCO}_{3}$ com formação de óxido de cálcio.

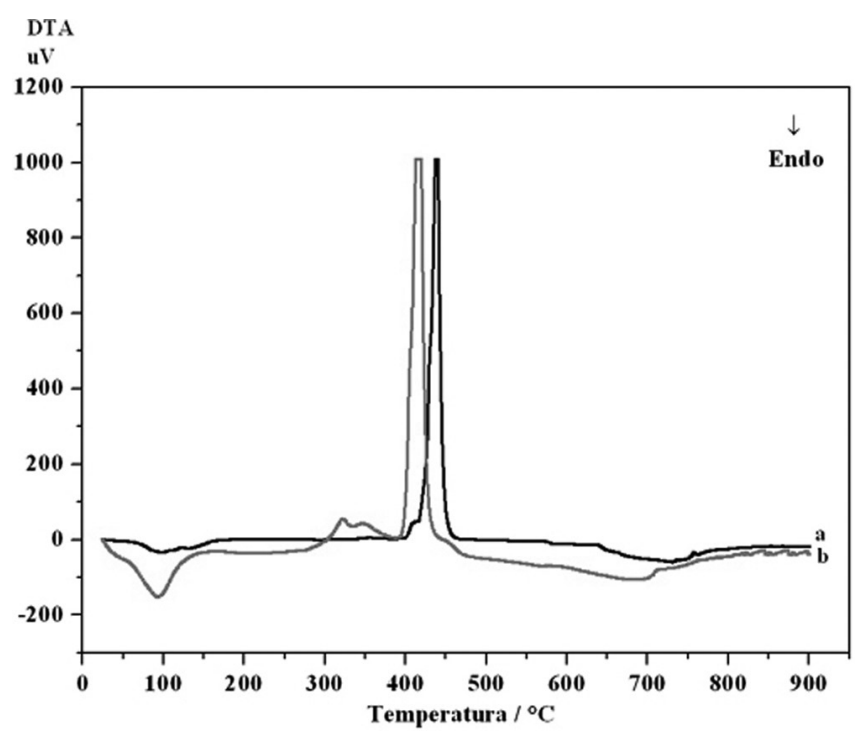

Figura 2. Curvas DTA do lactato de cálcio princípio ativo e da amostra do comprimido

Na curva DTA da amostra do comprimido, observa-se um evento endotérmico entre 59,4 e $123,2{ }^{\circ} \mathrm{C}$ característico do processo de desidratação, além de dois picos exotérmicos consecutivos entre 280,5 e $377,2^{\circ} \mathrm{C}$ relacionados, provavelmente, à decomposição dos excipientes presentes na amostra do comprimido. Entre 380,5 e $475,0^{\circ} \mathrm{C}$ é observado um pico exotérmico relacionado, provavelmente, à decomposição do lactato de cálcio para formação do $\mathrm{CaCO}_{3}$ e uma endoterma no intervalo de 600,7 a $720,5{ }^{\circ} \mathrm{C}$, indicativo da decomposição do $\mathrm{CaCO}_{3}$ e formação de $\mathrm{CaO}$.

A Figura 3 apresenta as curvas DSC do lactato de cálcio e da amostra do comprimido. Na curva DSC do lactato de cálcio podem-se observar dois eventos endotérmicos, o primeiro entre 37,8 e $100,2^{\circ} \mathrm{C}$ $\left(\mathrm{T}_{\mathrm{p}}=66,7^{\circ} \mathrm{C}\right) \mathrm{com} \mathrm{DH}=-150,3 \mathrm{~J} \mathrm{~g}^{-1} \mathrm{e}$ o segundo no intervalo entre 103,9 e $144,4{ }^{\circ} \mathbf{C}\left(\mathrm{T}_{\mathrm{p}}=118,6^{\circ} \mathrm{C}\right)$ e $\mathrm{DH}=-10,5 \mathrm{~J} \mathrm{~g}^{-1}$ relacionados à desidratação da amostra. Um terceiro evento endotérmico também está evidenciado nesta curva, na faixa de 313,9 a $368,8{ }^{\circ} \mathrm{C}$ $\left(\mathrm{T}_{\mathrm{p}}=342,9^{\circ} \mathrm{C}\right)$ com um valor de $\mathrm{DH}=-30,4 \mathrm{~J} \mathrm{~g}^{-1}$, relacionado à decomposição térmica da amostra.

A curva DSC da amostra de comprimido apresenta dois eventos endotérmicos, o primeiro no intervalo de 44,7 a $110,7^{\circ} \mathrm{C}$ 


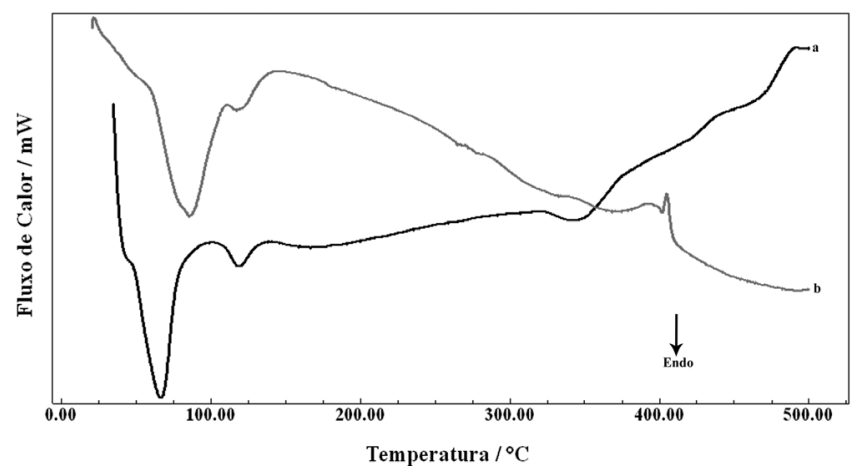

Figura 3. Curvas DSC de (a) lactato de cálcio e (b) amostra do comprimido

$\left(\mathrm{T}_{\mathrm{p}}=85,6^{\circ} \mathrm{C}\right)$ e $\mathrm{DH}=-308,7 \mathrm{~J} \mathrm{~g}^{-1}$ e o segundo no intervalo de 110,7 a $144,7{ }^{\circ} \mathrm{C}\left(\mathrm{T}_{\mathrm{p}}=117,1^{\circ} \mathrm{C}\right) \mathrm{com} \mathrm{DH}=-11,2 \mathrm{~J} \mathrm{~g}^{-1}$. Estes eventos podem ser atribuídos ao processo de desidratação de constituintes do comprimido, conforme observado na Figura 3. Um evento exotérmico também é observado entre 388,8 e $417,9{ }^{\circ} \mathrm{C}\left(\mathrm{T}_{\mathrm{p}}=404,9{ }^{\circ} \mathrm{C}\right)$ cujo $\mathrm{DH}=20,8 \mathrm{~J} \mathrm{~g}^{-1}$ e pode estar ligado à decomposição da amostra, para que haja a formação de $\mathrm{CaCO}_{3}$.

Os difratogramas de raios-X do lactato de cálcio (a) e da amostra do comprimido (b) estão mostrados na Figura 4. Os resultados indicam que o lactato de cálcio apresenta estrutura não cristalina. Em relação à amostra do comprimido foram encontrados picos indicativos de cristalinidade referentes à presença de talco $\left[\mathrm{Mg}_{6}\left(\mathrm{Si}_{2} \mathrm{O}_{5}\right)_{4}(\mathrm{OH})_{4}\right]$ utilizado como excipiente, na função de diluente.

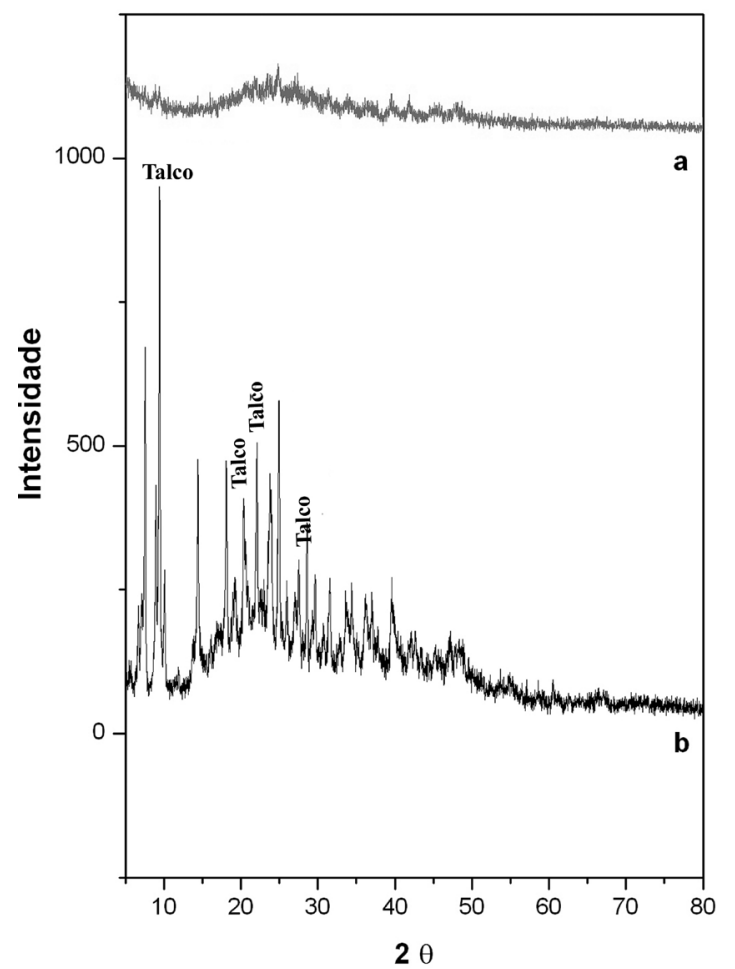

Figura 4. Difratogramas de raios $X$ de (a) lactato de cálcio e (b) amostra do comprimido

Na Figura 5 encontram-se os difratogramas de raios $\mathrm{X}$ dos intermediários obtidos por termogravimetria, a 550,0 ${ }^{\circ} \mathrm{C}$, para as amostras do lactato de cálcio (a) e da amostra de comprimido (b). Os difratogramas de ambas as amostras mostram picos característicos de carbonato de cálcio (JCPDF: 85-1108). ${ }^{12}$ Esses resultados estão de acordo com os obtidos a partir da terceira perda de massa da termogravimetria e sugerem que esse evento térmico de perda de massa é devido à formação principalmente de carbonato de cálcio. $\mathrm{O}$ difratograma de raios $\mathrm{X}$, obtido a partir da amostra do comprimido a essa temperatura, exibe também picos do talco de acordo com (JCPDF: 03-0881). ${ }^{12}$

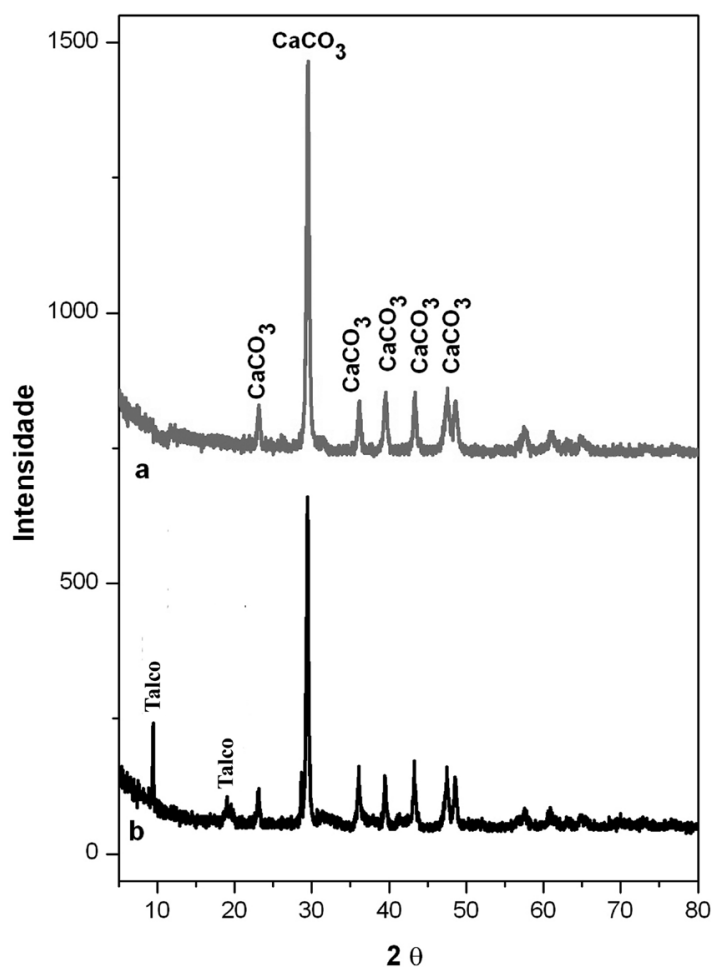

Figura 5. Difratogramas de raios X obtidos pela termogravimetria a $550{ }^{\circ} \mathrm{C}$ para (a) lactato de cálcio e (b) amostra do comprimido

Os difratogramas de raios $\mathrm{X}$ do resíduo obtido pela termogravimetria a $900,0^{\circ} \mathrm{C}$ para o lactato de cálcio (a) e a amostra do comprimido (b) estão apresentados na Figura 6. Verifica-se a presença de $\mathrm{Ca}(\mathrm{OH})_{2}$ nas duas amostras (JCPDF:81-2041) ${ }^{12}$ uma vez que na decomposição térmica do carbonato de cálcio ocorre a formação de óxido de cálcio e gás carbônico. No entanto, o $\mathrm{CaO}$ converte-se em hidróxido de cálcio em contato com a umidade do ar explicando, assim, a presença dos picos de hidróxido de cálcio nos difratogramas.

$\mathrm{Na}$ micrografia da amostra de lactato de cálcio, Figura $3 \mathrm{~S}$ do material suplementar, observa-se a presença de partículas angulares com granulometria da ordem de $150 \mu \mathrm{m}$ e na amostra do comprimido, Figura 4S do material suplementar, verificam-se partículas características do princípio ativo e agregados, devido à presença dos excipientes que fazem parte da formulação farmacêutica.

O teor de cálcio obtido por meio das curvas termogravimétricas pode ser determinado pela perda de massa referente à liberação de $\mathrm{CO}_{2}$ observada no último evento térmico, que consiste na decomposição térmica do $\mathrm{CaCO}_{3}$, com formação do $\mathrm{CaO} \cdot{ }^{13}$

$$
\mathrm{CaCO}_{3(\mathrm{~s})} \rightarrow \mathrm{CO}_{2(\mathrm{~g})}+\mathrm{CaO}_{(\mathrm{s})}
$$

Para efeito de comparação dos resultados do teor de cálcio obtido pelas curvas termogravimétricas, foram realizadas análises utilizando a espectrometria de emissão óptica com plasma indutivamente acoplado (ICP-OES). Esses dados também foram comparados com o valore teórico de cálcio contido na bula do medicamento. Os resultados obtidos, assim como o desvio-padrão e coeficiente de variação encontram-se na Tabela 2 . Todas as análises foram realizadas em triplicata. 
Tabela 2. Teores de cálcio em (\%) no comprimido à base de lactato de cálcio utilizando termogravimetria, ICP-OES e cálculos teóricos

\begin{tabular}{|c|c|c|c|c|c|c|c|c|}
\hline \multicolumn{3}{|c|}{ Termogravimetria } & \multicolumn{3}{|c|}{ Teórico } & \multicolumn{3}{|c|}{ ICP } \\
\hline \% cálcio 8,93 & $\begin{array}{l}\text { Desvio pa- } \\
\text { drão } 0,60\end{array}$ & $\begin{array}{c}\text { Coef. de } \\
\text { variação 6,60 }\end{array}$ & $\begin{array}{c}\text { \% cálcio } \\
13,14\end{array}$ & $\begin{array}{l}\text { Desvio pa- } \\
\text { drão } 0,26\end{array}$ & $\begin{array}{c}\text { Coef. de } \\
\text { variação 2,00 }\end{array}$ & \% cálcio 8,93 & $\begin{array}{l}\text { Desvio pa- } \\
\text { drão } 0,26\end{array}$ & $\begin{array}{c}\text { Coef. de } \\
\text { variação 2,90 }\end{array}$ \\
\hline
\end{tabular}

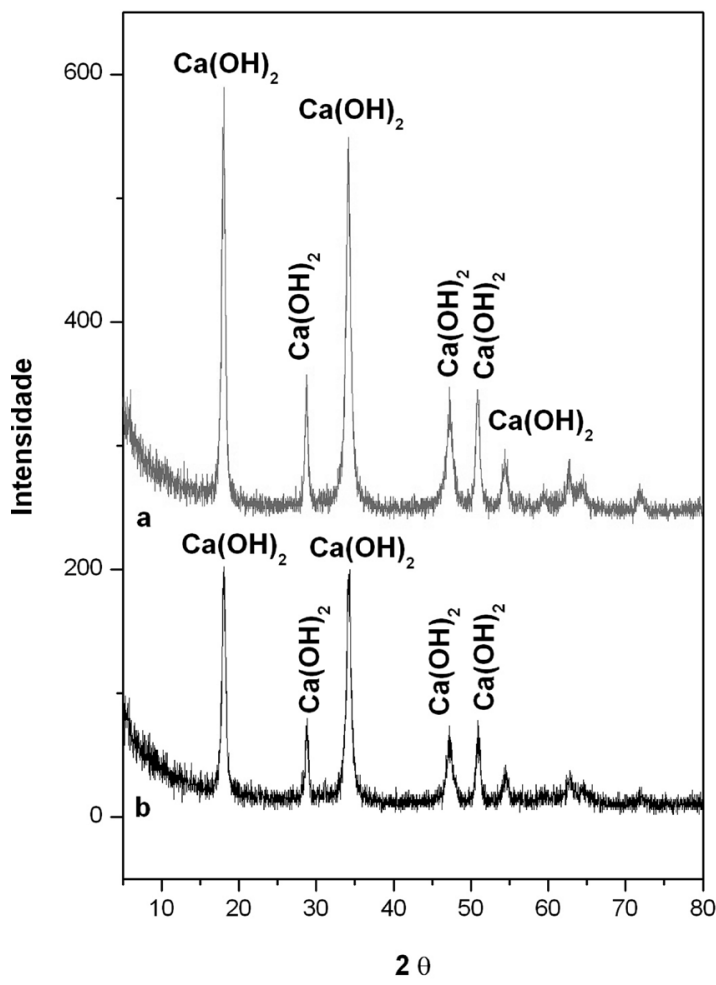

Figura 6. Difratogramas de raios $X$ dos resíduos obtidos por termogravimetria a $900{ }^{\circ} \mathrm{C}$

No medicamento analisado constam teoricamente $250 \mathrm{mg}$ de lactato de cálcio que, segundo cálculos teóricos, fornecem um teor de $13,14 \%$ de cálcio elementar. No entanto, os resultados obtidos pela termogravimetria e ICP-OES apresentaram um teor de $8,93 \%$ de cálcio na amostra do comprimido.

\section{CONCLUSÃO}

Os resultados obtidos por termogravimetria na determinação do teor de cálcio na amostra de comprimido à base de lactato de cálcio mostraram-se satisfatórios quando comparados aos obtidos por ICP-OES. Isso comprova que a termogravimetria pode ser utilizada na determinação do teor de cálcio em formulações farmacêuticas, pois a técnica oferece rapidez, economia no uso das amostras, eliminação do uso de reagentes ácidos no processo de abertura de amostra e eficiência nos resultados.

\section{MATERIAL SUPLEMENTAR}

O material suplementar (Figuras 1S a 4S) está disponível gratuitamente em http://quimicanova.sbq.org.br, na forma de arquivo PDF.

\section{AGRADECIMENTOS}

À Petrobras, Capes e ao Nupprar/UFRN.

\section{REFERÊNCIAS}

1. Fardellone, P.; Cotté, F. E.; Roux, C.; Lespessailles, E.; Mercier, F.; Gaudin, A. F.; Joint Bone Spine 2010, 77, 154.

2. Campos, L. M. A.; Liphaus, B. L.; Silva, C. A. A.; Pereira, R. M. R.; J. Pediatr. 2003, 79, 6.

3. http://portal.saude.gov.br/portal/aplicacoes/noticias/default. $\mathrm{cfm} ? \mathrm{pg}=\mathrm{dspDetalheNoticia \& id \_ area}=124 \&$ CO_NOTICIA $=10063$, acessada em Junho 2012.

4. Raymond, C. R. Em Handbook of Pharmaceuticals Excipients; Raymond, C. R; Sheskey, P. J.; Owen, S. C., eds.; $5^{\text {th }}$ ed., Pharmaceutical Press: London, 2006.

5. Castilho, L. S.; Paixão, H. H.; Perini, E.; Rev. Saúde Pública 1999, 33, 3.

6. Wendlandt, W. W.; Thermal Analysis, Willey: New York, 1986.

7. Wesolowski, M.; Konarski, T.; Anal. Chem. 1996, 41, 387.

8. Sakata, Y.; Shiraishi, S.; Otsuka, M.; Colloids Surf., B 2005, 46, 3.

9. Sakata, Y.; Shiraishi, S.; Takayama, K.; Otsuka, M.; Colloids Surf. B. 2006, 51, 2.

10. Pereira, J. G.; Okumura, F.; Ramos, L. A.; Cavalheiro, E. T. G.; Nóbrega, J. A.; Quim. Nova 2009, 32, 6.

11. Carvalho Filho, R. O.; Franco, P. I. B. M.; Conceição, E. C.; Leles, M. I. G.; J. Therm. Anal. Calorim. 2009, 97, 2.

12. Philips, A. B. V.; X'PERT HIGHSCORE; Program for Crystal Structure identification; Koninkliik e Philips Electronics N. V., Holanda, 2001.

13. Souza, S. P. M. C.; Morais, F. E.; Santos, E. V.; Martinez-Huitle, C. A.; Fernandes, N. S.; J. Therm. Anal. Calorim.(2011), doi 10.1007/s10973011-2119-z. 


\section{DETERMINAÇÃO DO TEOR DE CÁLCIO EM COMPRIMIDO À BASE DE LACTATO DE CÁLCIO UTILIZADO NO TRATAMENTO DA OSTEOPOROSE}

Sheila P. M. C de Souza, Francisca E. de Morais, Elisama V. dos Santos, Márcia L. da Silva, Carlos A. Martinez-Huitle e Nedja S. Fernandes*

Instituto de Química, Universidade Federal do Rio Grande do Norte, Av. Senador Salgado Filho, 3000, Campus Universitário, 59078-970 Natal - RN, Brasil

$$
\left[\underset{\mathrm{OH}}{\mathrm{CH}_{3}-\underset{1}{\mathrm{CH}}-\mathrm{C}_{-\mathrm{O}^{-}}^{=\mathrm{O}}}\left[\mathrm{Ca}_{2}^{2+}\right]\right.
$$

Figura 1S. Estrutura química do lactato de cálcio

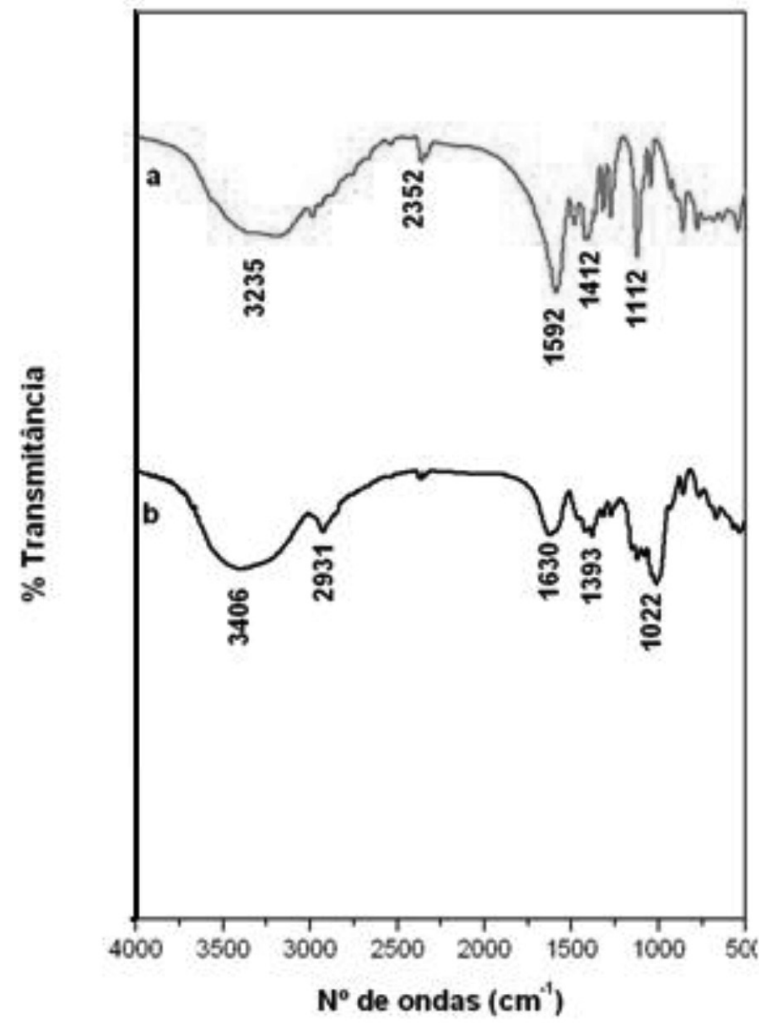

Figura 2S. Espectros de infravermelho do (a) lactato de cálcio (b) amostra de comprimido

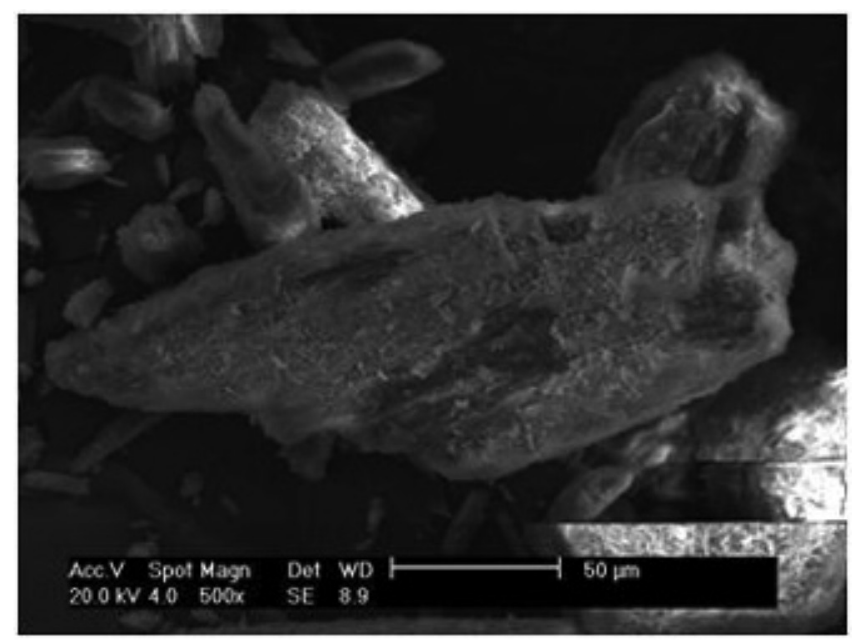

Figura 3S. Micrografia da amostra de lactato de cálcio obtida com aumento de $500 x$

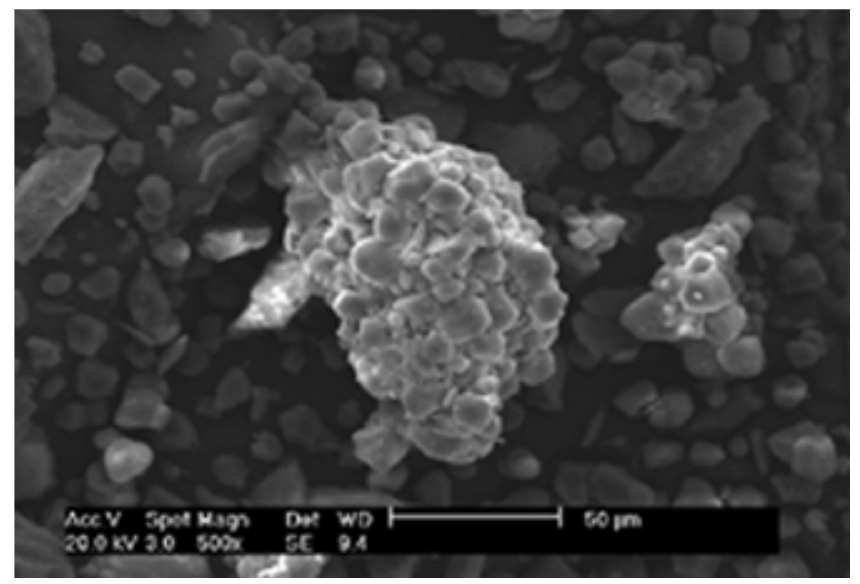

Figura 4S. Micrografia da amostra do comprimido obtida com aumento de $500 x$ 